\title{
GENERACIONES MÉDICAS DEL PERÚ Y EL ORIGEN DE LA UPCH
}

\section{INTRODUCCIÓN}

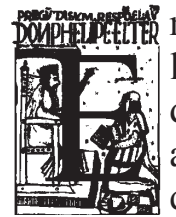

nulio de 1961, centenares de profesores de la Facultad de Medicina de la Universidad de San Marcos, renunciaron a sus cargos y acordaron conformar una nueva universidad que denominada al inicio "Universidad Peruana de Ciencias Médicas y Biológicas" pasó a llamarse "Universidad Peruana Cayetano Heredia" y continúa la tradición de rigor en la docencia y calidad en la investigación que caracterizó a la Facultad de Medicina.

Se ha escrito bastante sobre esta decisión singular en el Perú, donde la renuncia al cargo es excepcional y también admirable por la casi unanimidad de los profesores sanfernandinos que renunciaron a su claustro. El movimiento someramente descrito líderes: Alberto Hurtado y Honorio Delgado, y seguidores, valiosos profesores que dirigían cátedras y grupos de investigación; pero poco se ha tratado sobre que motivó a los centenares de profesores asociados, auxiliares y jefes de práctica, que siguieron sin vacilar a los profesores principales.

¿Cuál fue el cemento que les unió?, ¿Qué experiencias previas los marcaron para adoptar tal decisión?

Este artículo ofrece una nueva explicación a este fenómeno singular en el Perú y que ha devenido en una institución de excelencia que ahora se elogia unánimemente.

\section{GENERACIONES MÉDICAS EN PERÚ}

Javier Mariátegui, destacado psiquiatra y estudioso de la medicina peruana, trató el tema en la conferencia inaugural de las actividades de la Academia Nacional de Medicina en marzo del año 2000; la denominó "La Generación del Novecientos y la Medicina Peruana" y la inició expresando que:

"En el debate sobre la cultura peruana no han estado ausentes conspicuas figuras médicas del pasado y pocas en nuestro tiempo; luego señala como protagonista y cronista de la medicina peruana en la segunda mitad del siglo XIX a José Casimiro Ulloa, y en las primeras décadas del siglo XX a Hermilio Valdizán”. Continúa Mariátegui: "La generación novecentista volcó su espíritu de investigación sobre el reciente pasado republicano y sobre el presente del Perú, iniciando el análisis de los elementos formativos de su conciencia nacional, de sus instituciones, de sus hombres y de su proceso intelectual y social.

En esa generación novecentista destacan los médicos: Hermilio Valdizán, Julio C. Tello, Carlos Monge, Carlos Enrique Paz Soldán, Constantino Carvallo, Baltazar Caravedo Prado, y Sebastián Lorente de Patrón. La generación del Novecientos fue llamada también "arielista" por su coincidencia cronológica con la publicación del libro de Rodó sobre Ariel (1900), principalmente por su concepción elitista, y su intento de reintegrar culturalmente a Nuestra América con su pasado hispánico.

La continuidad del examen de la realidad nacional, fue tarea que se impusieron los integrantes de la Generación del Novecientos, tras la infausta Guerra del Pacífico, que tuvo como pensador representativo a Francisco García Calderón y conspicuos mílites entre los que se encontraba una docena de médicos de gran importancia en la historia, de los que sólo mencionaremos ahora a Hermilio Valdizán, quien es no sólo una figura representativa de la cultura peruana sino el fundador de la moderna Psiquiatría.

Añadió, que desde la perspectiva europea, Ulloa se ocupa de manera crítica de la evolución social y política de los primeros tramos del Perú republicano, con "condena del militarismo, de la intimidación de la prensa, del descuido ante la educación y ante el problema indígena", además de la conducción económica, la esterilidad del parlamento, la falta de independencia del poder judicial, que concluye con un llamado a la juventud "a favor de la libertad, la igualdad, el progreso social y la dignidad nacional". 
Como casi todos los "arielistas" de la Generación del Novecientos, Valdizán estaba influido por el positivismo y, a diferencia de otros que preconizaron el laicismo y el credo liberal, el maestro sanmarquino prefirió una postura más bien conservadora. Aunque alejado de la política partidaria, Valdizán fue no sólo mílite sino fundador del Partido Nacional Democrático de don José de la Riva Agüero. Otras figuras médicas novecentistas fueron también miembros de ese partido que no pasó de la etapa embrionaria de su gestación y primeros desarrollos: Carlos Monge M., Julio C. Tello (volcado pronto a la antropología), Francisco Graña Reyes, Augusto Dammert, Miguel Aljovín, Baltazar Caravedo Prado, Sebastián Lorente, Juan Voto Bernales, Jorge Morrison, Ricardo Palma Román, Enrique León García, Guillermo Gastañeta, Constantino J. Carvallo, Raúl Rebagliatti, Aníbal Corvetto, Julio César Gastiaburú, Ricardo Pazos Varela, fundadores de cátedras notables en la Facultad de San Fernando, creadores de las distintas especialidades.

A continuación expuso que "Los médicos de la generación novecentista representaron una élite" dominante de la profesión en el Perú. Como en ninguna otra generación médica, eran auténticos representantes de la "República Aristocrática". Todos se volcaron, en mayor o en menor medida, a la investigación de la realidad del país. Algunos fueron cabezas visibles de la escuela médica peruana y dieron curso a estirpes familiares notables".

El método que diseñó Ortega y Gasset para el estudio de las generaciones comienza por la búsqueda de la generación decisiva y de la figura epónima; creo que en el Perú y en el siglo XX la Generación es la del Centenario o de la Reforma Universitaria y la figura epónima, Alberto Hurtado, reformador de la Facultad de Medicina de la UNMSM (1956-1961) y fundador de la UPCH.

La generación del Centenario ha sido descrita y tratada por varios autores: Luis Alberto Sánchez, Jorge Basadre, Raúl Porras, pero centrada a ellos y sus compañeros en Letras o Derecho, C. Pacheco Vélez amplía la relación para incluir a algún médico, pero deja de lado a figuras tan importantes como Eleazar Guzmán Barrón, líder de los estudiantes de medicina, de quien dice Basadre con admiración es el representante del nuevo indio. "Eleazar Guzmán Barrón fue la más alta figura del movimiento juvenil de aquel año. $\mathrm{Su}$ brillante hoja de servicios como alumno, como médico y como hombre de ciencia. Eleazar símbolo del indio de mañana. He aquí a uno de los grandes peruanos del siglo XX"; explica su olvido el alejamiento de EGB del Perú, quien tuvo brillante trayectoria como profesor de bioquímica en la Universidad de Chicago; sin embargo, Guzmán Barrón mantuvo su interés en el país y fue autor del proyecto para contar en los años $50 \mathrm{~s}$, con un Consejo Nacional de Investigación y fue el gestor de la creación de las facultades de medicina de Arequipa y Trujillo, ambas tienen su busto en lugar destacado.

Pacheco Vélez cita en su relación del Centenario sólo a un médico neurólogo y muy distinguido personaje como fue Oscar Trelles, pero omite a figuras como Alberto Hurtado y Carlos Gutiérrez-Noriega, psiquiatra y farmacólogo tempranamente desaparecido, pero con importante obra que ha sido compilada recientemente.

En el Perú se llama Generación de Reforma Universitaria por el gravitante papel que en ella cumplieron algunos de sus miembros: Haya y los arriba mencionados; lo que no se ha comentado es que en la huelga de 1920 cerró la Facultad de Medicina de Lima, la única del país y obligó a varios de sus estudiantes a salir al exterior, donde se graduaron; Alberto Hurtado en la Universidad de Harvard, O. Soto, P. Weiss, P. Ga1lardo y otros en Madrid; creo que como consecuencia algunos de ellos fueron "vacunados" contra la reforma universitaria; de ello trata lo siguiente.

Retornados al Perú en los años 30s, la mayoría de estos médicos se incorporó a la Facultad de Medicina como jóvenes profesores con adecuada formación, pero de nuevo en 1933, la UNMSM fue clausurada por 


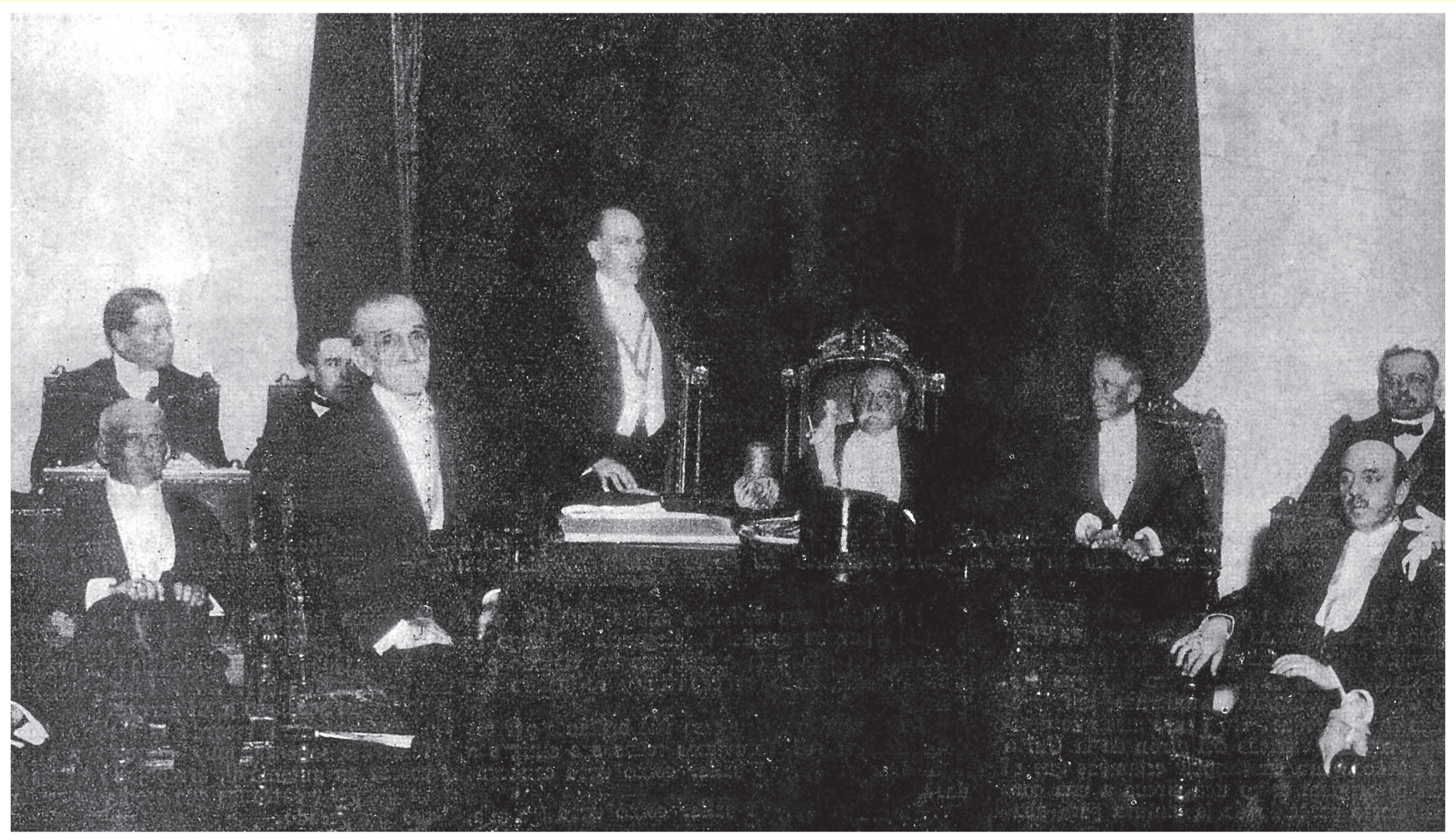

Apertura del Año Académico de 1922 de la Universidad Nacional Mayor de San Marcos.

En la FOto PUBlicAda EN LA REVISTA "Mundial", aparece El ReCTOR

Dr. Manuel Vicente Villarán haciendo uso de la palabra.

MANuel Vicente Villarán (1873-1958), conNotado JuRista y EducAdor

Se doctoró en Derecho en 1894 y de Abogado en 1896

Ministro de Justicia, Culto e Instrucción (1908), Decano del

Colegio de Abogados de Lima (1914) y de la Facultad de Derecho (1918)

Rector de la Universidad Nacional Mayor de San Marcos (1922)

Candidato a la presidencia de la República (1936) 
el gobierno y algunos de estos médicos se apartaron para dedicar su tiempo a la práctica privada.

No fue el caso de Alberto Hurtado, quien providencialmente recibió una beca de la Fundación Rockefeller que le permitió investigar la función respiratoria del hombre, durante tres años en la recién creada Facultad de Medicina de Rochester, con extraordinarias publicaciones en las más prestigiosas revistas científicas de EE.UU.

La UNMSM se reabrió en 1935 y su Facultad de Medicina se esforzó en mantener su calidad, mediante rigurosas pruebas de admisión de sus postulantes, que procedían de todo el país; dice Basadre que por años la Facultad de Medicina era la mejor de la UNMSM; en ella trabajaron varios de los médicos graduados en el extranjero en los años $20 \mathrm{~s}$, como Alberto Hurtado, Oscar Trelles, quien vino de Francia, Hugo Pesce de la Universidad de Génova, Pedro Weiss de Alemania, entre otros. Un número de los profesores de medicina de los 30s había cursado estudios de postgrado en Europa, como C. Krumdieck en París, R. Franco en la U. Pennsylvania, J. B. Lastres en varios países europeos.

Fue este grupo de médicos docentes el que sólo diez años después, en 1945 sufrió las consecuencias de otra Reforma Universitaria que, liderada por Luis Alberto Sánchez, decretó el libre ingreso de los postulantes a la Facultad de Medicina, la cual tuvo que recibir entre 1946 y 1948 un mil alumnos cada año, es decir diez veces el número habitual; esta improvisación temeraria alteró gravemente la enseñanza, a esto se agregó la tacha estudiantil a varios profesores en algunos casos "porque sus clases eran muy difíciles". Ello fue suprimido en 1949 cuando se abolió la Ley Universitaria y la Facultad de Medicina recuperó su exigencia en la admisión; era la tercera dosis de "vacuna anti reforma" que recibía el claustro de entonces.

Al retornar la democracia al Perú en 1956 con Manuel Prado, en el Congreso se preparó una nueva
Ley Universitaria que otorgaba un trato especial a las facultades de medicina (UNMSM, UNSA y UNT) así el artículo 34 decía que éstas, por la naturaleza de sus funciones, fijarían la proporción de alumnos que formaría parte del Consejo Universitario y Asamblea Universitaria, que para las restantes facultades debería ser de un tercio. De nuevo era rector de San Marcos, Luis Alberto Sánchez, quien además era senador aprista, en alianza con el pradismo.

Los estudiantes y también los profesores de otras facultades, reclamaron por este trato discriminatorio y el Congreso debatía las propuestas. Del lado de la Facultad de Medicina, el decano Hurtado, con el apoyo y solidaridad de la mayoría de los profesores defendían la situación y señalaban en pronunciamientos públicos, los precedentes funestos que la Facultad de Medicina sufrió, en el período 1945-1948.

Las autoridades y profesores de la Facultad de Medicina anunciaron que renunciarían a sus cargos; lo cual no fue creído por el gobierno, ni por la Universidad; al promulgarse la ley que suprimía el artículo 34 presentaron sus renuncias individuales 490 profesores de medicina al entonces rector J. L. Barandiarán, era yo un joven profesor y estuve presente en la entrega de los documentos a un sorprendido rector en su casa del distrito de Lince.

Las tres experiencias antes descritas y que he llamado "vacunas", habían cumplido su función y los profesores de medicina rechazaban lo que para ellos era nocivo y extraño.

La renuncia tiene siempre un sentimiento de pérdida y ése era el ambiente en el claustro pleno de la Facultad de Medicina en julio de 1961, fue allí que Víctor Alzamora Castro, emocionado al extremo leyó su propuesta para crear una universidad libre para enseñar e investigar y se conformó la Unión Médica de Docentes Cayetano Heredia, había nacido la nueva institución; esa noche falleció víctima de un infarto Víctor Alzamora Castro, el autor de la propuesta. 
La generación que estudió Medicina entre los años 20 y 40 tuvo destacados médicos que coincidían en señalar a Víctor Alzamora Castro, como el representante de un grupo que inició las grandes especialidades en el Perú, él fue un cardiólogo eminente, investigador original y escritor, sus coétaneos fueron varios de los profesores asociados al momento de renuncia a la U. San Marcos, entre ellos Carlos Peschiera, cirujano de tórax pionero; Fernando Cabieses, neurocirujano y escritor polifacético; fue Cabieses quien me refirió el aprecio que sus compañeros sentían por Alzamora, a quien veían como el sucesor de Hurtado en el decanato de la Facultad de Medicina.

Javier Arias-Stella ha descrito su generación en San Fernando a la que denomina "de la media centuria" (estudiaron medicina entre 1940 y 1960) y de la cual él es uno de los líderes, desde estudiante; luego patólogo investigador y político destacado, compañeros suyos son Uriel García, Carlos Roe y Juan Serkovic, entre otros.

En uno de sus últimos artículos "Los del 50 desde el siglo XXI" Luis Jaime Cisneros, recién desaparecido, dice: "El tema de la generación del 50 (de cuyos integrantes he sido y sigo siendo amigo) me interesa, en la perspectiva que puede atraer a un filólogo, diarios y revistas de la época, libros accesibles en Lima o traídos por viajeros; la Democracia Cristiana y Acción Popular; el gobierno militar, las unidades escolares ¿Qué pensaban los arquitectos (Fernando Belaúnde y su revista), los que no eran escritores? La inquietud en Medicina, que lleva a la creación de la Universidad Cayetano Heredia... El interés por la sociología y la lenta transformación de las universidades... De repente descubrimos que si colocamos a los más conocidos en su estricta perspectiva, la generación cobra nueva interpretación y nuevo horizonte. Necesitamos reflexionar sobre esto, pero añado una pregunta, que todavía carece de una respuesta clara. ¿Esa generación es el futuro que previó la generación anterior? ¿O es la ruptura, el rechazo total, la negación del pasado y el anuncio de un anhelo todavía no bien fundado (o no bien esclarecido)".
Recapitulando lo antes descrito, creo que la Generación Centenario o de la Reforma, se dividió en dos grupos: los estudiantes de medicina que al cierre de la UNMSM en los años veinte salieron al extranjero para proseguir estudios y los de letras y derecho que permanecieron en el país y fueron a las Universidades de Cusco y Trujillo, que seguían ofreciendo clases.

Al retorno de los médicos, varios fueron incorporados como profesores a San Fernando, comenzaron en su carrera docente, tenían posiciones directivas a fines de los años 50; fueron ellos los que respaldaron a Hurtado, primero en la defensa de los privilegios de la Facultad y luego en la renuncia masiva de julio de 1961 que llevó a la creación de la UPCH.

Esta interpretación de lo ocurrido entre 1920 y 1960 se refuerza con lo descrito para el corto período 1945-1948, en el rectorado de Luis Alberto Sánchez y el decanato de Sergio Bernales.

La revisión de las actas del Consejo de Facultad (faltan varias de sesiones críticas) que he realizado, describen los debates y luchas entre la mayoritaria representación estudiantil aprista y los contados que no lo eran, que luego destacaron como profesores.

\section{GENERACIONES DE DIRECTIVOS E INVESTIGADORES EN LA UPCH}

Considerando la propuesta de Ortega y Gasset de 15 años como intervalo generacional, en los 50 años que cumple la UPCH deben apreciarse por lo menos tres generaciones.

$\begin{array}{lcc} & \text { Nacidos } & \text { Vigencia } \\ \text { 1. Fundadores médicos } & 1900-1920 & 1961-1976 \\ \begin{array}{l}\text { 2. Continuadores científicos } \\ \text { 3. Directivos médicos }\end{array} & & \\ \quad \text { y científicos } & 1936-1935 & 1976-1990 \\ \end{array}$




\section{Fundadores médicos}

Elijo esta denominación, porque fue característica inicial de la UPCH su excelencia en la docencia e investigación médicas, explicada por la calidad y entrega de los profesores e investigadores que renunciaron a San Fernando y prosiguieron sus tareas en la nueva universidad; destacan entre ellos los tres primeros rectores: Delgado, Hurtado y Monge; y profesores como Oscar Trelles, iniciador de la neurología en el Perú y Pedro Weiss, médico y antropólogo, con discípulos como Javier Arias-Stella y Uriel García Cáceres.

\section{Continuadores científicos}

En esta generación herediana ubicó a los profesores e investigadores que tuvieron cargos directivos entre 1976 a 1990, todos ellos fueron médicos pero dedicaron su mayor esfuerzo a la Facultad de Ciencias y a los Institutos. Destacan en este grupo Alberto Cazorla, primer decano de la nueva facultad, Ramiro Castro de la Mata, quien también la dirigió; Enrique Fernández, director del Programa Académico de Ciencias y Hugo Lumbreras, fundador y director del Instituto de Medicina Tropical; me ubico en este grupo cuando dirigí el
Instituto de Investigaciones de la Altura por los años setenta y asumí el rectorado en 1989-1994.

\section{Médicos y científicos}

Dirigieron la UPCH y sus institutos de Altura y Medicina Tropical entre 1991 al presente; todos estudiaron en la UPCH; en el grupo están Humberto Guerra Allison y Eduardo Gotuzzo del Instituto de Medicina Tropical, Gustavo Gonzáles Rengifo y Fabiola León-Velarde del Instituto de Investigaciones de la Altura, todos se reconocen como discípulos de la generación anterior.

Es interesante notar que entre los miembros de estas tres generaciones de rectores y decanos de la $\mathrm{UPCH}$, hubo relación de maestro-alumno.

Esta situación es única en el país; una universidad creada en base al profesorado de una Facultad de Medicina con devoción y calidad en la investigación, que es dirigida desde hace cincuenta años por investigadores y docentes con relación discipular, que han mantenido la excelencia inicial y es hoy reconocida internacionalmente como una universidad de investigación.

¿Cómo hacer para que ello continúe? 